\title{
Molecular evaluation of 7 sexually transmissible microorganisms in symptomatic and asymptomatic Italian childbearing age women: is Ureaplasma parvum a real innocent bystander?
}

\author{
Manuela Avolio, Maria Luisa Modolo, Paola Stano, Rita De Rosa, Alessandro Camporese \\ Clinical Microbiology and Virology Laboratory, Department of Laboratory Medicine, Hospital of \\ Pordenone, Italy
}

\begin{abstract}
Summary
Background: Symptoms of most common bacterial and parasitic sexually transmitted infections tend to be non-specific and typically have a variety of different potential causal agents that may require different treatments. In this field the pathogenic potential of genital Ureaplasma species is still uncertain and debated. The goal of this study was to investigate the prevalence of Chlamydia trachomatis (CT), Neisseria gonorrhoeae (NG), Trichomonas vaginalis (TV), Mycoplasma genitalium (MG), Mycoplasma hominis (MH), Ureaplasma urealyticum (UU) and Ureaplasma parvum (UP) in a cohort of symptomatic and asymptomatic childbearing age women and to assess the relationships between bacterial vaginosis and symptoms with both UU and UP.

Materials and Methods: DNA of 2735 endocervical specimens was consecutively analysed by a commercial multiplex real-time polymerase chain reaction for detection of 7 multiple target sequences simultaneously: CT, NG, TV, MG, MH, UU and UP.

Results: Out of the total number of population studied $(\mathrm{n}=2735), \mathrm{UP}$ was found to be the species with highest prevalence (30.9\%) followed
\end{abstract}

\footnotetext{
Correspondence: Manuela Avolio, Clinical Microbiology and Virology Laboratory, Department of Laboratory Medicine, Hospital of Pordenone, via Montereale 24, Pordenone, Italy.

Tel: +39.0434 .399044 . Fax: +39.0434 .399170 .

E-mail: manuela.avolio@aas5.sanita.fvg.it
}

Key words: Ureaplasma parvum; Mycoplasma; Ureaplasma; Sexually transmitted diseases; Multiplex Real Time PCR.

Contributions: the authors contributed equally.

Conflict of interest: the authors declare no potential conflict of interest.

Received for publication: 4 March 2016.

Revision received: 5 May 2016.

Accepted for publication: 7 May 2016.

(C) Copyright M. Avolio et al., 2016

Licensee PAGEPress, Italy

Microbiologia Medica 2016; 31:5864

doi: $10.4081 / \mathrm{mm} .2016 .5864$

This article is distributed under the terms of the Creative Commons Attribution Noncommercial License (by-nc 4.0) which permits any noncommercial use, distribution, and reproduction in any medium, provided the original author(s) and source are credited. by MH (6.5\%), UU (6.3\%), CT (2.6\%), MG (0.8\%) and TV (0.9\%). UP single species detection was extremely significant in symptomatic women with normal flora $(\mathrm{P}<0.0001)$. The correlation of UP in symptomatic women with bacterial vaginosis was not significant $(\mathrm{P}=0.3387)$.

Conclusions: Our results suggest a potential specific etiological role to UP, still considered rightly or wrongly innocent bystander, despite the lack so far of specific-species culture tests.

\section{Introduction}

Symptoms of most common bacterial and parasitic sexually transmitted infections (STIs) tend to be non-specific and typically have a variety of different potential causal agents that may require different treatments. Between some of the sexual transmissible pathogens Chlamydia trachomatis (CT), Neisseria gonorrhoeae (NG), Trichomonas vaginalis (TV), Mycoplasma genitalium (MG) are established pathogens of STIs in women genital tract, while the role of Mycoplasma hominis (MH), Ureaplasma urealyticum (UU), and Ureaplasma parvum (UP) as genital tract pathogens is still strongly debated (5). Typical symptoms of STIs as vaginal discharge, itching or odour are also associated with bacterial vaginosis (replacement of the vaginal flora by an overgrowth of anaerobic bacteria including Prevotella spp, Mobiluncus spp., Gardnerella vaginalis, Ureaplasma spp., Mycoplasma spp., and numerous fastidious or uncultivated anaerobes), trichomoniasis, and candidiasis (5). The major symptoms of cervicitis are a mucopurulent endocervical exudate and sustained endocervical bleeding (5). However, many infections are asymptomatic, thus clinicians should perform appropriate diagnostic testing $(2,4,26)$.

Ureaplasma spp. are known to colonize mucosal surfaces of the lower urogenital tract in healthy people (24). Risk factors for colonization include multiple sexual partners, low socioeconomic status and oral contraception. UU (previously known as UU biovar 2) and UP (previously known as UU biovar 1) are commonly found in the human urogenital tract (40-80\% of sexually active women). Horizontal transmission of Ureaplasma spp. is by sexual contact and genital infection is usually asymptomatic (16). Because of the frequency with which UU occurs in healthy asymptomatic individuals, it has been suggested that only certain subgroups of the species are disease-associated $(9,14,15,20,24)$. Some clinical cases of several urogenital and systemic diseases, including non-gonococcal urethritis (NGU), endometritis, chorioamnionitis, abortion, birth of premature babies, septic arthritis, bacteraemia and meningitis are reported in the literature to be related to these microorganisms $(2,19,22,24)$. However, the pathogenic potential of genital Ureaplasma spp. in infections of the female reproductive tract and par- 
ticularly their role in the female infertility is still uncertain and debated $(12,23,24,26)$. The confounding factors are not only the high prevalence of these microorganisms in asymptomatic persons, but also the inability of the traditional culture-based methods, widely used for their detection, to distinguish between the two species of the genus Ureaplasma among UP and UU (24). It is important to stress that before UU and UP were recognized as separate species, they were both designated UU, making interpretation of the results of previous studies difficult (26).

Ureaplasma spp., known as strains difficult to culture in the laboratory, are very small such as $10 \pm 5 \mathrm{~m}$ diameter and have not a cell wall. When culturing in the laboratory, infection is judged through $\mathrm{pH}$ change in liquid culture using such principle. But recently, as studies have shown that polymerase chain reaction (PCR) method is more sensitive, PCR method is preferred to classical culture (14). When determining the clinical significance of ureaplasma infection, the differentiation of colonization and infection is necessary because of the high prevalence of Ureaplasma spp. in the healthy population. The recent introduction of various PCR-based methods, has improved the overall diagnostic yield of ureaplasma detection because of their high sensitivity and ability to discriminate between the two Ureaplasma spp., compared to traditional culture-based methods. The detection of STIs using multiplex-based methods able to detect simultaneously several microorganisms distinguishing different Ureaplasma spp., might be helpful to clinicians to prescribe appropriate antibiotics for patients according to the results.

The aim of this study was to investigate by multiplex real time PCR the prevalence of seven sexually transmissible microorganisms (UP, UU, MH, MG, CT, TV and NG) in a cohort of Italian childbearing age women and to asses the relationships between bacterial vaginosis and symptoms with both UU and UP for the first time distinguishable at the species level by a new commercial molecular method.

\section{Materials and Methods}

\section{Study population and clinical specimens}

We retrospectively evaluated 2735 outpatients childbearing age women come to our observation from December 2012 to June 2014. All were consecutive women attended to Clinical Microbiology and Virology of Pordenone Hospital to get a vaginal and endocervical swabs for a routine screening in absence of symptoms $(n=919)$ or for the presence of symptoms of genital infections as vaginal discharge, vaginal itching, pelvic pain, burning sensation or pain during urination or sexual activity $(n=1816)$. The vaginal and endocervical swabs were collected by medical microbiologists, who followed the regular procedures for speculum examination and used manufactured collection kits (Copan Italia SpA, Brescia, Italy).

\section{Bacterial culture and microscopic examination}

Vaginal swabs were executed for $\mathrm{pH}$ determination, microscopic examination and standard cultures (aerobic and anaerobic bacteria and yeasts). Endocervical swabs were executed for CT, NG, TV, Mycoplasma spp., Ureaplasma spp. molecular detection and for NG cultures (26).

Liquid Amies Elution Swabs (Copan Italia SpA, Brescia, Italy) was used to collect and transport vaginal samples for solid culturing of common bacteria according to laboratory-defined standard procedures. Nugent method was used to diagnose bacterial vaginosis. Gram-stained smears were evaluated by the Nugent scoring system $(17,26)$. The Nugent method is a standardized method, designed to evaluate bacterial vaginosis. A Nugent score from 0 to 3 indicates a normal flora; from 4 to 6 is called intermediate state, and from 7 to 10 indicates bacterial vaginosis. Bacterial vaginosis is a modification of the vaginal flora characterized by a diminished or absent flora of lactobacilli, which increases the vaginal $\mathrm{pH}(10,17,18)$.

\section{Molecular investigations}

Flocked swabs and Universal Transport Medium (Copan Italia SpA, Brescia, Italy) were used to collect and transport endocervical samples for molecular investigations.

The MICROLAB Nimbus IVD system was used for the nucleic acid automated extraction (Hamilton, Reno, NV, USA) to maximize the workflow and accuracy. Real-time PCR amplification was performed using the Anyplex ${ }^{\mathrm{TM}}$ II STI-7 Detection Assay (Seegene, Seoul, Korea), in accordance with the manufacturer's protocol, in a CFX96 real- time thermocycler (Bio-Rad, Hercules, CA, USA). The Anyplex ${ }^{\text {TM }}$ II STI-7 Detection is a novel multiplex real-time PCR assay that permits the simultaneous amplification, detection and differentiation of target nucleic acids of CT, NG, MG, MH, UU, UP, TV and Internal Control (IC) $(3,6,13)$. Anyplex ${ }^{\mathrm{TM}}$ II STI-7 Detection Assay is based on a newly developed TOCETM technology, which makes it possible to detect multipathogens in a single fluorescence channel on real-time PCR instruments. In current melting curve analysis, temperature differences are often observed among DNA that has high sequence variation, resulting in issues in clinical diagnostic fields where accurate and reproducible test results are critical. However, TOCE ${ }^{\mathrm{TM}}$ technology is designated to be not affected by sequence variations; therefore guaranteeing consistent Tm values. The Anyplex ${ }^{\mathrm{TM}}$ II STI-7 Detection represents a new class of molecular tests by cyclic-Catcher Melting Temperature Analysis (CMTA) that are multiplexed. The cyclic-CMTA method can discriminate major pathogen in the co-infected samples. In PCR, efficiency can be reduced by inhibitors that may be present in the clinical specimens. An IC is incorporated into the product as an exogenous whole process control in order to monitor nucleic acid isolation and to check for possible PCR inhibition. The IC is coamplified with the target nucleic acids within the clinical specimens.

Anyplex $^{\text {TM }}$ II STI-7 shows detection limit for sensitivity of 10 copies/reaction for CT and NG ( $<5.710$ copies/mL), and 50 copies/reaction for UU, UP, MG, MH, TV ( $<28.550$ copies $/ \mathrm{mL}$ ).

Amplirun Ureaplasma urealyticum DNA (Vircell Microbiologists, Spain) was used as quantitative control.

\section{Statistical analysis}

Statistical analysis were performed using a $\chi 2$ test. Results with $\mathrm{P}<0.05$ were considered to be statistically significant.

\section{Results}

Cohort characteristics and distribution of microorganisms detected: 208 out of 2735 patients examined were $<25,1016$ were $25-35$ and 1511 were $<35$ years old. Of patients examined, 546 out of 2735 were pregnant; 1816 out of 2735 were symptomatic and 919 asymptomatic; 852 out of 2735 were positive for Candida albicans (in $35 \%$ of cases in association with at least one of the other microorganisms studied). Table 1 shows the cohort characteristics (age, pregnancy, presence or absence of symptomatology, vaginal candidiasis) and the associated negative or positive results, included the correlation with each single species detected. Of samples 1418 out of 2735 (51.8\%) were negatives; 1317 out of 2735 (48.2\%) had at least one of seven microorganism targets in her genital tract. In this study NG was never detected and MG was always in association with other organisms. Out of the total number of population studied ( $\mathrm{n}=2735), 845$ were positive for UP (30.9\%), 173 for UU (6.3\%), 178 for MH (6.5\%), 23 for MG (0.8\%), 72 for CT (2.6\%), 26 for TV (0.9\%) (Table 2). 
Among the single microorganism detections, 521 out of 628 (82.9\%) of patients with UP were symptomatic versus 54 out of 87 (62.1\%), 23 out of $26(88.5 \%)$ and 8 out of $9(88.8 \%)$ with UU, MH, CT respectively. In this study MG and TV were never detected in asymptomatic women.

As shown in Figure 1, UP was the species more often detected alone, with a prevalence of $28.7,11.6$ and $17.8 \%$ in symptomatic, asymptomatic and pregnant subgroups respectively.

\section{Mycoplasma spp. and Ureaplasma spp.: correlation with symptoms and microscopic features}

Change in vaginal flora (normal flora or absence of lactobacilli) and clinical features (presence or absence of symptoms) and its relationship with Mycoplasma spp. and Ureaplasma spp. were also studied (Table 3). To rule out that these changes were related to other pathogens present, we included in the statistical analysis only the single species determination. Of samples, 517 out of 764 (67.7\%) positives for Mycoplasma spp. and Ureaplasma spp. were classified by Nugent score as normal vaginal flora, 247 out of 764 (32.3\%) as absence of lac- tobacilli. UP were found more often from women with normal flora $(\mathrm{P}=0.025)$ while $\mathrm{MH}$ and $\mathrm{MG}$ were found more often from women with absence of lactobacilli ( $\mathrm{P}=0.05$ and $\mathrm{P}<0.001$, respectively).

Of samples, 621 out of 764 (81.3\%) positives for Mycoplasma spp. and Ureaplasma spp. were symptomatic, 143 out of 764 (18.7\%) were asymptomatic. UP and MG were found more often from symptomatic patients $(\mathrm{P}=0.01$, and $\mathrm{P}=0.01$ respectively), while $\mathrm{UU}$ was found more often from women with absence of symptoms $(\mathrm{P}<0.001)$. No significant differences were found for $\mathrm{MH}$ and change in vaginal flora $(\mathrm{P}=0.33)$.

In Table 3, the subgroup indicated as Absence of Lactobacilli includes patients with bacterial vaginosis (Nugent score $>6$ and $\mathrm{pH}>4.5$ ); the subgroup Normal Flora includes patients with Nugent score from 0 to 6 and $\mathrm{pH}$ 3.5-4.5. We observed, as expected $(10,17,18)$, a correlation between $\mathrm{pH}$ and Nugent class (data not shown).

In Table 4, the subgroup indicated as Symptomatic with Normal flora (SN) was the more representative of women with UP single species detection (54.7\%). No difference between SN and Asymptomatic with Normal flora subgroups in patients with single UU detection was observed (36 and 38\%).

Table 1. Distribution of no, single and multiple infection by age, clinical features, vaginal candidiasis and pregnancy.

\begin{tabular}{|c|c|c|c|c|c|c|c|c|c|c|c|c|c|c|c|c|}
\hline \multirow[t]{2}{*}{$\begin{array}{l}\text { Cohort characteristics } \\
(n=2735)\end{array}$} & \multicolumn{2}{|c|}{$\begin{array}{l}\text { Negatives } \\
(n=1418)\end{array}$} & \multicolumn{2}{|c|}{$\begin{array}{c}\text { UP } \\
(n=628)\end{array}$} & \multicolumn{2}{|c|}{$\underset{(n=87)}{\text { UU }}$} & \multicolumn{2}{|c|}{$\begin{array}{c}\text { MH } \\
(n=26)\end{array}$} & \multicolumn{2}{|c|}{$\begin{array}{c}\mathrm{MG}^{*} \\
(\mathrm{n}=23)\end{array}$} & \multicolumn{2}{|c|}{$\begin{array}{c}\text { CT } \\
(n=9)\end{array}$} & \multicolumn{2}{|c|}{$\begin{array}{c}\text { TV } \\
(n=2)\end{array}$} & \multicolumn{2}{|c|}{$\begin{array}{l}\text { Multiple } \\
\text { infections } \\
(\mathrm{n}=565)\end{array}$} \\
\hline & $\mathbf{N}$ & $\%$ & $\mathbf{N}$ & $\%$ & $\mathbf{N}$ & $\%$ & $\mathbf{N}$ & $\%$ & $\mathbf{N}$ & $\%$ & N & $\%$ & $\mathbf{N}$ & $\%$ & N & $\%$ \\
\hline \multicolumn{17}{|l|}{ Age } \\
\hline$<25(\mathrm{n}=208)$ & 100 & 7.1 & 48 & 7.6 & 7 & 8.1 & 2 & 7.7 & 2 & 8.7 & 1 & 11.2 & 0 & 0 & 50 & 8.8 \\
\hline $25-35(\mathrm{n}=1016)$ & 407 & 28.7 & 263 & 41.9 & 41 & 47.1 & 10 & 38.5 & 13 & 56.5 & 4 & 44.4 & 2 & 100 & 289 & 51.2 \\
\hline$>35(\mathrm{n}=1511)$ & 911 & 64.2 & 317 & 50.5 & 39 & 44.8 & 14 & 53.8 & 8 & 34.8 & 4 & 44.4 & 0 & 0 & 226 & 40 \\
\hline Symptomatic $(\mathrm{n}=1816)$ & 701 & 49.4 & 521 & 82.9 & 54 & 62.1 & 23 & 88.5 & 23 & 100 & 8 & 88.8 & 2 & 100 & 507 & 89.7 \\
\hline Asymptomatic $(\mathrm{n}=919)$ & 717 & 50.6 & 107 & 17.1 & 33 & 37.9 & 3 & 11.5 & 0 & 0 & 1 & 11.1 & 0 & 0 & 58 & 10.3 \\
\hline Positive for $C$. albicans $(\mathrm{n}=852$ ) & 432 & 30.5 & 174 & 27.7 & 25 & 28.7 & 9 & 34.6 & 9 & 39.1 & 3 & 33.3 & 1 & 50 & 199 & 35.2 \\
\hline Pregnant $(n=546)$ & 353 & 24.9 & 97 & 15.4 & 15 & 17.2 & 2 & 7.7 & 0 & 0 & 0 & 0 & 0 & 0 & 79 & 13.4 \\
\hline
\end{tabular}

*was always in association with other organisms. UP, Ureaplasma parvum; UU, Ureaplasma urealyticum; MH, Mycoplasma hominis; MG, Mycoplasma genitalium; CT, Chlamydia trachomatis; TV, Trichomonas vaginalis; $\mathrm{NG}$, Neisseria gonorrhoeae.

Table 2. Single and multiple species detection and percentage of prevalence by each microorganism detected.

\begin{tabular}{lccccccc} 
& UP & UU & MH & NG & CT & TV \\
Single microrganisms detected $(\mathrm{n}=752)$ & 628 & 87 & 26 & & 9 & 2 & 0 \\
Multiple microrganisms detected $(\mathrm{n}=565)$ & 217 & 86 & 152 & 23 & 63 & 24 & 0 \\
\hline Total number of positive samples $(\mathrm{n}=1317)$ & 845 & 173 & 178 & 23 & 72 & 26 & 0 \\
Prevalence (\%) & 30.9 & 6.3 & 6.5 & 0.8 & 2.6 & 1.0 & 0 \\
\hline
\end{tabular}

UP, Ureaplasma parvum; UU, Ureaplasma urealyticum; MH, Mycoplasma hominis; MG, Mycoplasma genitalium; CT, Chlamydia trachomatis; TV, Trichomonas vaginalis; NG, Neisseria gonorrhoeae.

Table 3. Ureaplasma/Mycoplasmas single species (n=764): relationship with vaginal flora and clinical features.

\begin{tabular}{|c|c|c|c|c|c|c|}
\hline & $\begin{array}{l}\text { Normal flora } \\
(n=517), n(\%)\end{array}$ & $\begin{array}{c}\text { Absence of } \\
\text { lactobacilli } \\
(\mathrm{n}=247), \mathrm{n}(\%)\end{array}$ & P-value & $\begin{array}{c}\text { Symptomatic } \\
(\mathrm{n}=621), \mathrm{n}(\%)\end{array}$ & $\begin{array}{l}\text { Asymptomatic } \\
(\mathrm{n}=143), \mathrm{n}(\%)\end{array}$ & P-value \\
\hline UP $(n=628)$ & $436(84.3)$ & $192(77.7)$ & 0,025 & $521(83.8)$ & $107(74.5)$ & 0.01 \\
\hline UU $(\mathrm{n}=87)$ & 64 (12.3) & $23(9.3)$ & 0.211 & $54(8.7)$ & $33(23.0)$ & 0.001 \\
\hline $\mathrm{MH}(\mathrm{n}=26)$ & $13(2.5)$ & $13(5.2)$ & 0.05 & $23(3.7)$ & $3(2.1)$ & 0.33 \\
\hline $\mathrm{MG}^{*}(\mathrm{n}=23)$ & $4(0.7)$ & $19(7.7)$ & $<0.001$ & $23(3.7)$ & 0 & 0.01 \\
\hline
\end{tabular}

*was always in association with other organisms. UP, Ureaplasma parvum; UU, Ureaplasma urealyticum; MH, Mycoplasma hominis; MG, Mycoplasma genitalium. 
The presence of UP single species detection was extremely significant in symptomatic women with normal flora respect UU $(\mathrm{P}<0.0001)$. The correlation of UP in symptomatic women with absence of lactobacilli was not significant $(\mathrm{P}=0.3387)$ (Table 4$)$.

\section{Discussion and Conclusions}

Using a novel multiplex PCR assay we demonstrated that out of the seven sexual transmissible pathogens UP is the most frequently detected species, as stated in other studies (11). We also shown that the majority of UP infections were symptomatic and symptomatic infection

Table 4. Ureaplasma parvum and Ureaplasma urealyticum single detection in symptomatic and asymptomatic women.

\begin{tabular}{lccc} 
& $\begin{array}{c}\text { Single Ureaplasma species detection } \\
\text { UP }(n=628), \\
n(\%)\end{array}$ & $\begin{array}{c}\text { PU -value }(n=87), \\
n(\%)\end{array}$ & \\
Symptomatic & $521(82.9 \%)$ & $54(62.1 \%)$ & $<0.0001$ \\
Asymptomatic & $107(17 \%)$ & $33(37.9 \%)$ & \\
\hline SA & $177(28 \%)$ & $23(25 \%)$ & 0.3387 \\
AA & $15(2 \%)$ & 0 & \\
\hline SN & $344(55 \%)$ & $31(36 \%)$ & $<0.0001$ \\
AN & $92(15 \%)$ & $33(38 \%)$ & \\
\hline
\end{tabular}

SA, symptomatic+absence of lactobacilli; AA, asymptomatic+absence of lactobacilli; SN, symptomatic+normal flora; AN, asymptomatic+normal flora. was more likely with UP than with UU. In contrast with other studies $(7,21)$ in our cohort no significant differences were found for UU and change in vaginal flora $(\mathrm{P}=0.211)$, neither for $\mathrm{UU}$ and symptoms. Respect to UU, the presence of UP single species detection was extremely significant in symptomatic women with normal flora, thus suggesting a specific pathogenic role of UP, not linked to a concomitant status of bacterial vaginosis.

The present study has the following main strengths and limitations: i) this is the biggest cohort of symptomatic and asymptomatic subjects screened for genital infections by multiplex real time PCR directly in clinical sample; ii) to our knowledge this is the first study to evaluate simultaneously the prevalence in female Italian population of seven STIs-pathogens (UP,UU, MH, MG, CT, TV, NG) directly in clinical sample and to correlate the results to clinical and microscopic features; iii) on the basis of our knowledge this is the first study to describe the prevalence of MG in the Italian population directly on a heterogeneous cohort of patients.

On the other hand the different size of the symptomatic group and the asymptomatic group, due to consecutive patient recruitment, is a limitation of this study. This study does not involve a clinical-therapeutic implication as it is limited by its observational nature. Although Anyplex ST7 does not provide information on the antimicrobial susceptibility of $\mathrm{MH}$ and Ureaplasma spp., as standard culture method, it is important to underline that actually, except for MG, any indication to treat Mycoplasma spp. and Ureaplasma spp. exists, and that only about male urethritis treatment procedures for these organisms are reserved for situations in which these infections are suspected (e.g., urethral lesions, or severe dysuria and meatitis, which might suggest genital herpes) or when NGU is not responsive to recommended therapy $(2,5,25)$. However, macrolides and tetracyclines are still effective in treatment of ureaplasma infections $(7,11)$. Molecular-based technolo-

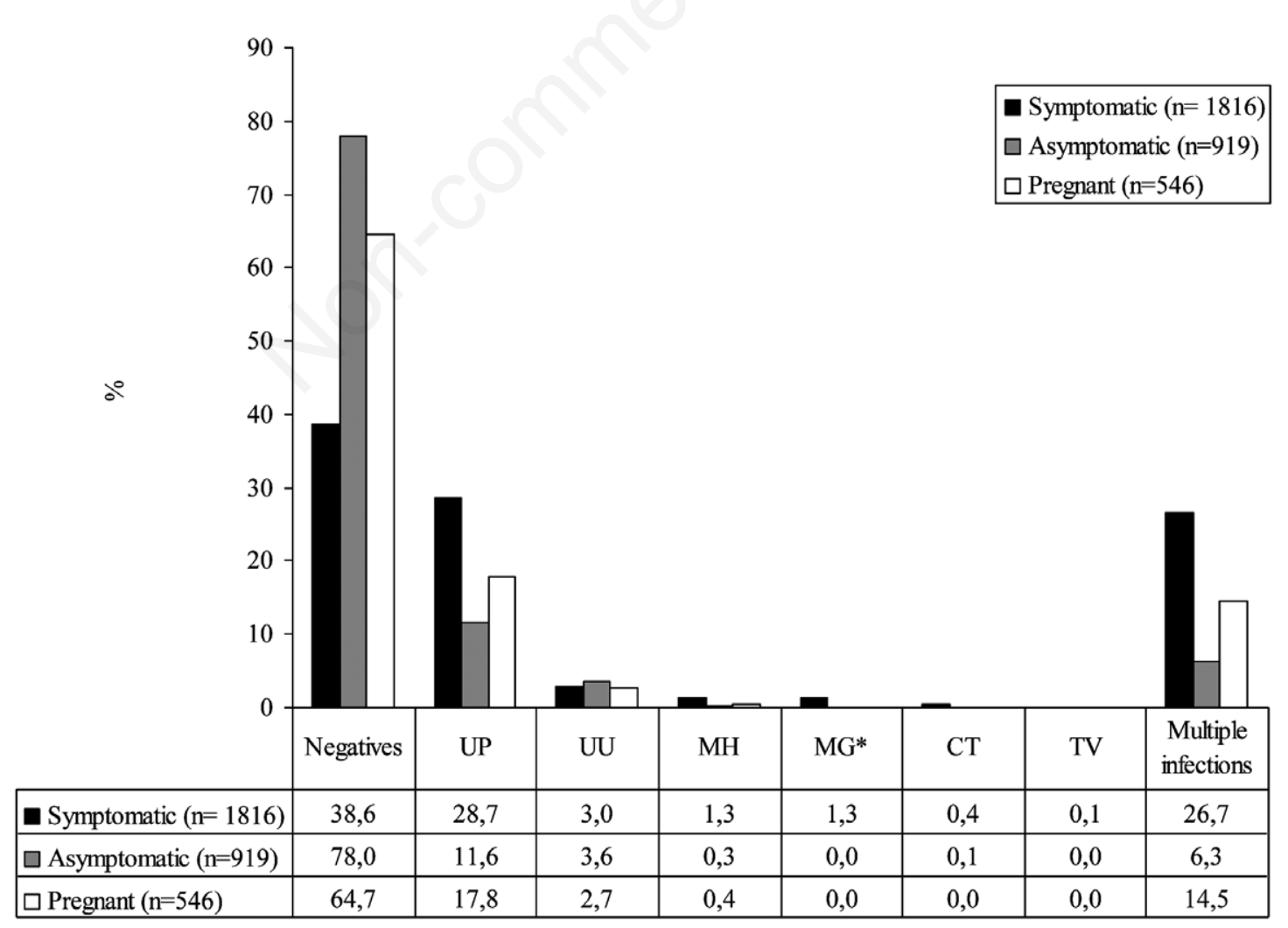

Figure 1. Single and multiple infections: distribution by clinical features. (MG* was always in association with other organisms). 
gies have several limitations (i.e. costs and the need for special equipment), which negatively affect the implementation of these techniques for routine laboratory diagnostics. More recently, the multiplex PCR assay has made it convenient for clinicians in many clinical fields to test for multiple causative organisms simultaneously. The multiplex PCR assay is a cost-effective diagnostic test because it allows for faster detection and a reduction in labour and reagent costs $(1,8)$. The development of completely automated systems (from extraction to read-out) may overcome this limitation.

In conclusion our results show the high prevalence of UP in endocervical specimens of symptomatic patients. We highlight the potential specific etiological role of UP, still considered rightly or wrongly an innocent bystander despite the lack so far of specific-species culture tests.

\section{References}

1. Avolio M, Diamante P, Modolo ML, et al. Direct molecular detection of pathogens in blood as specific rule-in diagnostic biomarker in patients with presumed sepsis - Our experience on a heterogeneous cohort of patients with signs of infective SIRS. Shock 2014;42,86-92.

2. Baron EJ, Miller JM, Weinstein MP, et al. A guide to utilization of the microbiology laboratory for diagnosis of infectious diseases: 2013 Recommendations by the Infectious Diseases Society of America (IDSA) and the American Society for Microbiology (ASM)(a). Clin Infect Dis 2013;57:e22-e121.

3. Berçot B, Amarsy R, Goubard A, et al. Assessment of coinfection of sexually transmitted pathogen microbes by use of the Anyplex II STI-7 molecular kit. J Clin Microbiol 2015;53;991-3.

4. Bradshaw C, Tabrizi S, Read T, et al. Etiologies of nongonococcal urethritis: bacteria, viruses, and the association with orogenital exposure. J Infect Dis 2006;193:336-45.

5. CDC. Sexually Transmitted Diseases Treatment Guidelines; 2015. Available from: http://www.cdc.gov/mmwr/pdf/rr/rr6403.pdf

6. Choe HS, Lee DS, Lee SJ, et al. Performance of AnyplexTM II multiplex real-time PCR for the diagnosis of seven sexually transmitted infections: comparison with currently available methods. Int $\mathbf{J}$ Infect Dis 2013;17:1134-40.

7. De Francesco MA, Negrini R, Pinsi G, et al. Detection of Ureaplasma biovars and polymerase chain reaction-based subtyping of Ureaplasma parvum in women with or without symptoms of genital infections. Eur J Clin Microbiol Infect Dis 2009;28:641-6.

8. Dierkes C, Ehrenstein B, Siebig S, et al. Clinical impact of a commercially available multiplex PCR system for rapid detection of pathogens in patients with presumed sepsis. BMC Infect Dis 2009; 9:126.

9. Gwee A, Curtis N. Ureaplasma-Are you sitting comfortably? J Infect 2014;68:19-23.

10. Hemalatha R, Ramalaxmi BA, Swetha E, et al. Evaluation of vaginal pH for detection of bacterial vaginosis. J Med Res 2013;138:354-9.
11. Hunjak B, Sabol I, Vojnovi G, et al. Ureaplasma urealyticum and Ureaplasma parvum in women of reproductive age. Arch Gynecol Obstet 2014;289:407-12.

12. Kasprzykowska U, Elias J, Elias M, et al. Colonization of the lower urogenital tract with Ureaplasma parvum can cause asymptomatic infection of the upper reproductive system in women: a preliminary study. Arch Gynecol Obstet 2014;289:1129-34.

13. Kim SJ, Lee DS, Lee SJ. The prevalence and clinical significance of urethritis and cervicitis in asymptomatic people by use of multiplex polymerase chain reaction. Korean J Urol 2011;52:703-8.

14. Kim Y, Kim J, Lee KA. Prevalence of sexually transmitted infections among healthy Korean women: implications of multiplex PCR pathogen detection on antibiotic therapy. J Infect Chemother 2014; 20:74-6.

15. Kong F, James G, Ma Z, et al. Phylogenetic analysis of Ureaplasma urealyticum-support for the establishment of a new species, Ureaplasma parvum. Int J Syst Bacteriol 1999;49:1879-89.

16. McCormack W. Urethritis. In: Mandell GL, Bennett JE, Dolin R, eds. Mandell, Douglas, and Bennett's principles and practice of infectious diseases. $7^{\text {th }}$ ed. Amsterdam: Elsevier Inc; 2010.

17. Nugent R, Krohn MA, Hillier SL. Reliability of diagnosing bacterial vaginosis is improved by a standardized method of gram stain interpretation. J Clin Microbiol 1991;29:297-301.

18. Schwebke JR, Hillier SL, Sobel JD, et al. Validity of the vaginal gram stain for the diagnosis of bacterial vaginosis. Obstet Gynecol 1996;88:573-6.

19. Sung TJ. Ureaplasma urealyticum or Ureaplasma parvum: what's the difference? Korean J Pediatr 2013;56:474-6.

20. Teng LJ, Zheng X, Glass JI, et al. Ureaplasma urealyticum biovar specificity and diversity are encoded in multiple-banded antigen gene. J Clin Microbiol 1994;32:1464-9.

21. Vancutsem E, Faron G, Foulon W, et al. Genital tract colonization with Ureaplasma spp. and its association with abnormal vaginal flora. J Med Microbiol 2015;64:654-6.

22. Viscardi RM. Ureaplasma species: role in diseases of prematurity. Clin Perinatol 2010;37:393-409.

23. Vouga M, Greub G, Prod'hom G, et al. Treatment of genital mycoplasma in colonized pregnant women in late pregnancy is associated with a lower rate of premature labour and neonatal complications. Clin Microbiol Infect 2014;20:1074-9.

24. Waites KB, Taylor-Robinson D. Mycoplasma and Ureaplasma. In: Versalovic J, Carrol KC, Funke G, Jorgensen JH, Landry ML, eds. Manual of clinical microbiology. $10^{\text {th }}$ ed. Washington DC: ASM Press; 2011. pp 974-85.

25. WHO. Guidelines for the management of sexually transmitted infections. 2003. Available from: http://applications.emro.who.int/ aiecf/web79.pdf

26. WHO. Laboratory diagnosis of sexually transmitted infections, including human immunodeficiency virus. 2013. Available from: http://apps.who.int/iris/bitstream/10665/85343/1/9789241505840_e ng.pdf 